\title{
Model of market equivalent of inter-system parallel exchange
}

\author{
Maksymilian Przygrodzki ${ }^{1,2^{*}}$, Pawet Kubek $^{1,2}$, and Rafat Gwoźdź ${ }^{1}$ \\ ${ }^{1}$ PSE Innowacje Sp. z o.o., Research and Development Department, 02-305 Warszawa \\ Al. Jerozolimskie 132, Poland \\ ${ }^{2}$ Technical Silesian University, Power System and Control Institute, 44-100 Gliwice \\ ul. B. Krzywoustego 2, Poland
}

\begin{abstract}
Equivalent of system interconnections is required for purpose of technical and economical analyses. The paper describes the author's market equivalent of inter-system parallel exchange. This approach allows to separation of import / export of power resulting from loop flows and demand profile. The presented model will be provided with the results of the NPS (Poland's National Power System) power flow analysis obtained using the built equivalent.
\end{abstract}

\section{Grid development process}

The process of planning the transmission network development is an activity whose aim is to assess the current state of the network and prepare prospective network structures taking into account both economic and technical issues (criteria). As a result of this process, a schedule of material actions is obtained, the implementation of which is to ensure fulfilment of the set objective. The conducted calculation activities are to lead to such solutions, the implementation of which will ensure reliable and economical operation of the power system in the future [1]. Therefore, the process of development planning brings with it the implementation of two threads: technical and economic (market).

Planning in the technical dimension covers the area of analysis, the primary objective of which is to examine the development needs of the transmission network due to the fulfilment of the set technical criteria. These criteria in the long-term horizon of time and the corresponding types of analysis include:

- power and energy adequacy - balance analysis, examining the possibilities of covering the existing demand;

- long-term thermal capacity of branches (lines and transformers) - power flow analyses, examining the load level in relation to the nominal (design) parameters. The results of these analyses determine the degree of network utilization and indicate the potential locations of future exceedances of the permissible branches' load capacities;

- short-circuit load capacity of branches - short-circuit analyses, examining the level of currents and short-circuit powers as well as defining development needs in the scope of devices and station requirements. Based on the results of these analyses, it is possible to

*Corresponding author: maksymilian.przygrodzki@pse.pl 
determine the scope of activities for adjusting the existing equipment to future loads in the disturbance states;

- the level of power reliability - reliability analyses, examining the structural reliability indicators shaped by the network topology, mutual location of sources and consumers, and unit reliability indicators of network elements, and then the effect of potential network failures on the transmission congestions.

As a result of technical analyses, network models including investment tasks are developed. These models are the basis for the concept of extending and modernizing the transmission network. The result of the adequacy analyses are guidelines regarding the requirements of the development of the generation sector, ensuring long-term energy security.

On the basis of the above, it should be noted that the technical dimension of planning gives guidelines for the formulation of development tasks and the maximum time-task framework for the security of implementation of development plans. Nevertheless, due to the scope of the research subject, which is the power system, a number of simplifying assumptions are applied, concerning both: the modelling of the network and the search for analytical solutions.

For example, technical and economic development analyses of selected areas require inter-system connections to be equivalented. In the further part of the article the authorial model of inter-system exchange on the synchronous cross-section of the National Power System (NPS) is described allowing for the separation of import / export of power resulting from the loop flows and from the demand profile. The presented model will be provided with the results of the flow analysis obtained using the built equivalent.

\section{Network equivalence}

Electric power systems often work as parallel connected systems. This applies to systems of different countries, different operators or different areas (voltage, geography, technology). Operation security requires then coordination of activities at the technical and managerial (operator) level. An example of such cooperation is the combined power system of Central and Western European countries, which includes, among others, NPS. Exploitation of large connected systems, as well as work on the planning of their development, necessitate the implementation of a series of analytical calculations regarding technical network analysis in the component areas [1]. Numerical calculations are possible only if reliable data describing the model of the analysed network is available. This model should take into account the topology of the network and the electrical parameters of its individual elements. However, there are some limitations regarding the preparation of data for very large network systems in technical analyses related to flow calculations or network development planning. The current model of the NPS along with the replacement model of neighbouring systems is very extensive, moreover, the complexity of the structure of the studied network plays a significant role due to the time and quality of the conducted analyses. The process of data preparation used in the calculation model is usually computer aided, however, a number of difficulties arise mainly due to the size of the computational task and the duration of numerical calculations.

A possible solution to the problem described above is the use of the so-called reduced models for the analysed networks [2]. The main advantage of using reduced models is reducing the size of the analysed network and limiting the level of detail of the description of some of its elements. The above factors simplify the stage of preparation of the calculation model, however it should be remembered that the applied reduction techniques should lead to obtaining a model describing the analysed network with acceptable accuracy. 
In the analyses of steady states of HV and EHV transmission networks, it is often necessary to replace a large part of the network with a smaller fragment (equivalent) in order to reduce the size of the tasks being solved [3]. From the numerical point of view, the equivalence of the network means the elimination of a certain group of network nodes using an admittance network model. The selection of nodes designated for elimination consists in defining the area of the network that is being considered. This area is usually designated by nodes to be eliminated and branches connected with them (including neighbouring nodes to eliminated nodes). For such a designated area, the node admittance matrix is calculated, and then the next nodes are eliminated according to the Gaussian elimination relationship [4]:

$$
Y_{i j}^{(n)}=Y_{i j}-\frac{Y_{i k} Y_{k j}}{Y_{k k}},
$$

in which $k$ is the eliminated node, while $i, j$ are indices of the remaining nodes (adjacent to eliminated nodes and nodes selected for elimination). Replacement network is obtained on the basis of the received node admittance matrix $Y_{\text {new }}$.

Analysis of the formula (1) results in the following conclusions:

- if nodes $i$ and $j$ are not adjacent to the eliminated node $k$, then during the elimination of $k$ node the admittance corresponding to these nodes does not change,

- due to the elimination of $k$ node, the network is spread in the form of a complete polygon on all its neighbour nodes, which replaces all connections of this node,

- own admittances of neighbour nodes of $k$ node are also modified according to the formula (1) for $i=j$.

The above conclusions are illustrated in Fig. 1.

a)

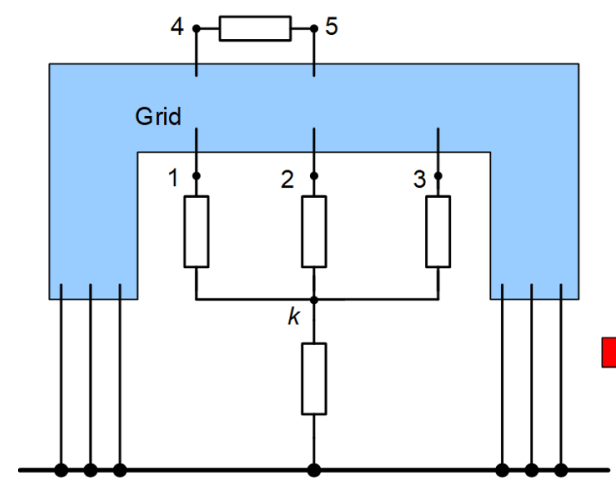

b)

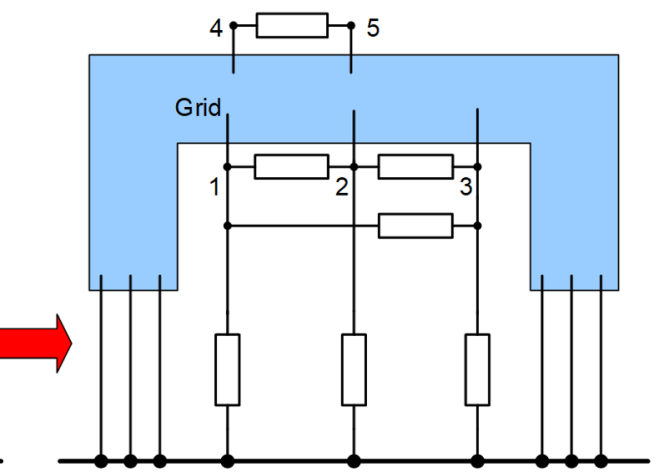

Fig. 1. Elimination of $k$ node; pre-elimination system (a); post-elimination system (b).

In the above figure, nodes $1,2,3$ are adjacent to the eliminated node $k$. Hence, after the elimination, a complete polygon is created on them. Nodes 4, 5 are not neighbours of node $k$, so their connection is not changed.

The presented idea of equivalence used in the NPS's development analyses allowed to reduce the analytical model. The effects of introducing an equivalent are shown in Table 1. The presented quantitative results refer to three models being the next reduction steps: 
- basic model covering the transmission and $110 \mathrm{kV}$ network,

- model after reduction of connectors (open couplings are eliminated, whereas closed couplings are treated as permanently galvanically connected, creating one busbar system) and elimination of block transformers, which results in permanent connection of generators directly to station nodes,

- model with an equivalent of $110 \mathrm{kV}$ distribution network.

In the model with the equivalent of $110 \mathrm{kV}$ network it is assumed:

- reduction of couplings in the $110 \mathrm{kV}$ network, similar to the full system,

- leaving the nodes in the model, which are connected to 3 or more branches (due to generation units not centrally dispatched - nJWCD),

- creating an equivalent of transient and tapping nodes and their aggregation to neighbouring nodes,

- liquidation of block transformers.

Table 1. Comparison of the number of elements of considered network systems depending on the adopted degree of model simplification.

\begin{tabular}{|c|c|c|c|}
\hline & Basic model & $\begin{array}{c}\text { Model without } \\
\text { couplings and block } \\
\text { transformers }\end{array}$ & $\begin{array}{c}\text { Model with 110 kV } \\
\text { network equivalent }\end{array}$ \\
\hline $\begin{array}{c}\text { Branches number, } \\
\text { including: }\end{array}$ & 5855 & 3979 & 2242 \\
\hline Lines number & 5239 & 3640 & 1903 \\
\hline Transformers number & 616 & 339 & 1377 \\
\hline Nodes number & 4620 & 3034 & 2050 \\
\hline Loads number & 4119 & 3942 & 1152 \\
\hline Generators number & 1152 & 1152 & 339 \\
\hline
\end{tabular}

The use of the $110 \mathrm{kV}$ network equivalent in the NPS model has a significant impact on reducing the number of nodes and branches. For example, the introduction of this equivalent results in a reduction in the number of nodes by 1657 (3243 nodes less in comparison to the basic system), and in the case of branches the difference is 1737 (3613 branches less in comparison to the basic system). The use of reduced systems allows for a significant acceleration of numerical calculations, while maintaining the required accuracy of the obtained results.

\section{Inter-system exchange with NPS}

The need to build equivalents of complex systems results not only from the fact of simplifying the internal structure of the examined system, but also from the environment in which the system operates [5]. In this approach, the goal may be to preserving the scale and directions of cross-border exchange, in particular on unsteered, i.e. synchronous cross- 
sections. For the comparison, the operator is decisive for non-synchronous cross-sections of the size and direction of the exchange.

In the analysis of the NPS work, it is important to preserve the mapping of inter-system exchange between Poland (NPS) and neighbouring systems on individual cross-sections: synchronous and non-synchronous.

The synchronous section includes interconnections with the power systems of Germany, the Czech Republic and Slovakia, through which the NPS operates synchronously with the area of Continental Europe. In the current power transmission studies, reference models are used: summer peak, summer valley, winter peak, winter peak, winter night valley. These systems include the generation correction in centrally dispatched generating units (JWCD) for maintaining the pre-set exchange balance on the synchronous cross-section throughout the analysis period.

In the NPS physical connections included in the synchronous cross-section include lines:

- with a rated voltage of $400 \mathrm{kV}$ :

- Mikułowa - Hagenwerder c.1;

- Mikułowa - Hagenwerder c.2;

- Dobrzeń - Albrechtice;

- Wielopole - Nosovice;

- Krosno - Lemesany c.1;

- Krosno - Lemesany c.2;

- with a rated voltage of $220 \mathrm{kV}$

- Krajnik - Vierraden t.1;

- Krajnik - Vierraden t.2;

- Kopanina - Liskovec;

- Bujaków - Liskovec.

The non-synchronous cross-section of the Polish Power System includes connections with the power systems of Sweden, Ukraine and Lithuania, in particular:

- HVDC cable at the Poland - Sweden connection;

- Poland - Ukraine connection at $220 \mathrm{kV}$ voltage level;

- Poland - Lithuania Back-To-Back connection at the $400 \mathrm{kV}$ voltage level.

It is worth mentioning that the non-synchronous section may include single $110 \mathrm{kV}$ lines on the Poland-Czech Republic cross-section, which feed consumers extracted in the Czech power system (hard coal mines, steelworks, municipal customers). The $220 \mathrm{kV}$ line between Zamość and Dobrotwór on the eastern border of Poland operates similarly. This line imports power from dedicated blocks of the Dobrotwór power plant.

Examples of values and directions of inter-system exchange in a graphical way are presented in Figure 2. The figure shows the set values on the non-synchronous crosssection, whereas for the synchronous cross-section the result of power exchange was marked as variable. This result depends on the balance of connected commercial areas and the technical parameters of the connected systems. Therefore, the synchronous crosssection becomes a natural contribution to the construction of the cross-border exchange equivalent for the NPS. 


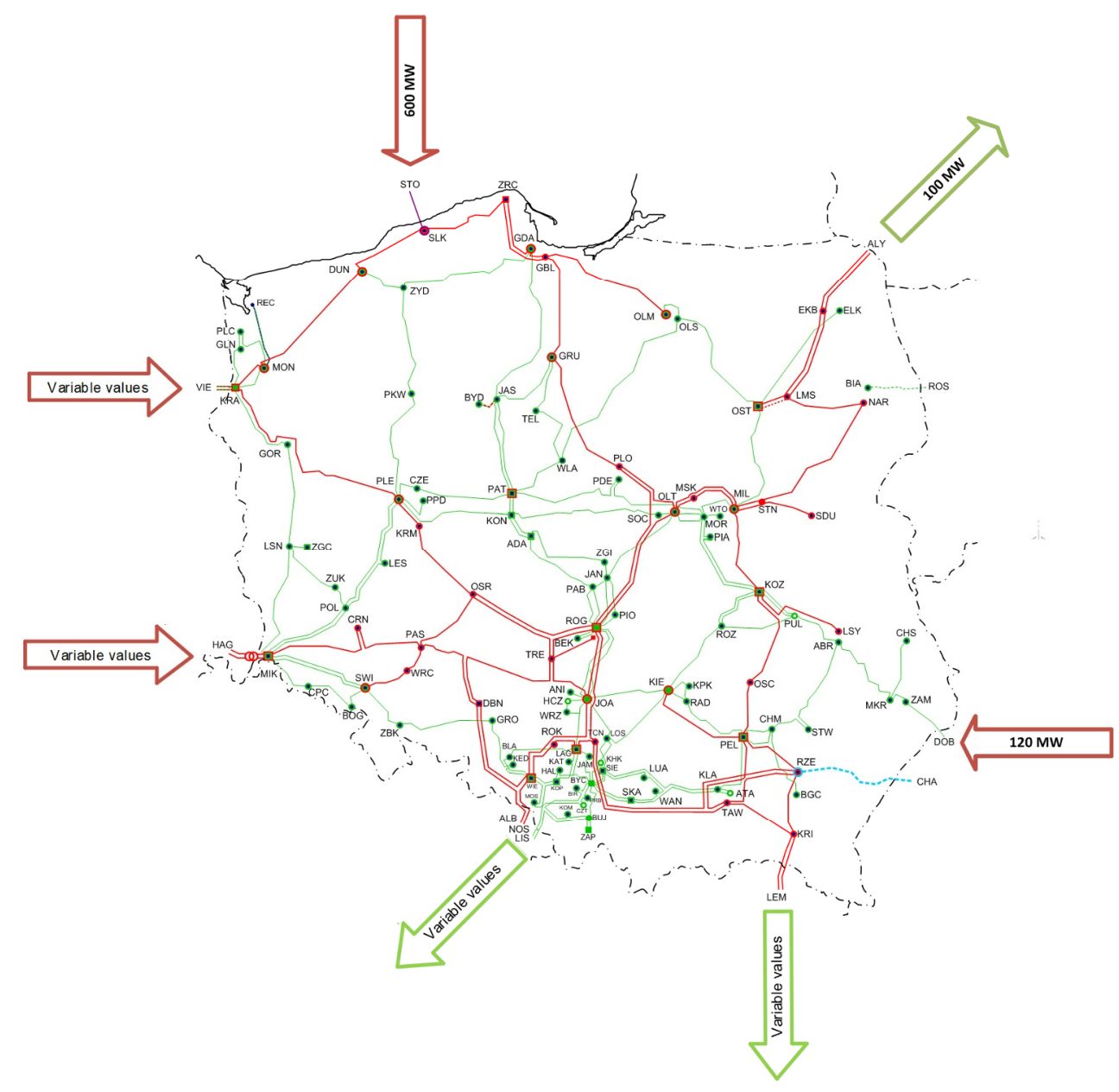

Fig. 2. Potential instantaneous values and directions of inter-system exchange in the NPS.

\section{Trade equivalent of inter-system exchange}

By limiting the size of the NPS model used for long-term analyses in the field of assessment of network connections loading and trade possibilities, a trade equivalent was developed for inter-system exchange [6]. In the first stage, the focus was on obtaining the assumed balance of inter-system exchange. The balance is close to value of $0 \mathrm{MW}$ on the synchronous cross-section representing the so-called loop flow. This flow is the result of high renewable generation in neighbouring power systems with high internal impedance of the network [7]. As a result, the flow direction leads to the load on the cross-section of exchange and the use of the NPS network for power transit [3].

In the next stage, in order to enable the introduction of cross-border exchange between Poland and neighbouring countries (import / export) in the calculations, an additional generator and additional load were modelled, to which an accordingly forecasted generation profile and demand profile were added. When modelling power imports, the generator is switched on, and in the case of export, the load is used.

The generator and load were assigned the coefficients (shift factors) [8] for distributing the cross-border exchange power to individual foreign network nodes determined on the 
basis of the NPS models (in KDM format) adopted as reference. These coefficients were determined in accordance with the principles described in Chapter 2.

The distribution coefficients determined for summer and winter conditions are presented in Table 2. These coefficients refer to both generated (import) and received (export) power in the foreign network equivalent. Figure 3 shows the model of interconnections of foreign network equivalent on a synchronous cross-section. The red and green colours correspond respectively to the grid voltages of $400 \mathrm{kV}$ and $220 \mathrm{kV}$. The model layer associated with cross-border exchange was mapped in blue.

Table 2. Values of distribution coefficients for summer and winter conditions.

\begin{tabular}{|c|c|c|c|}
\hline Connection & Node & Summer & Winter \\
\hline \multirow{2}{*}{ Germany } & zDV & 0.3 & 0.4 \\
\cline { 2 - 4 } & zDH & 0.7 & 0.6 \\
\hline \multirow{2}{*}{ Czech Republic } & zCA & 0.1 & 0.2 \\
\cline { 2 - 4 } & zCN & 0.5 & 0.5 \\
\hline Slovakia & zSK & 0.4 & 0.3 \\
\hline
\end{tabular}

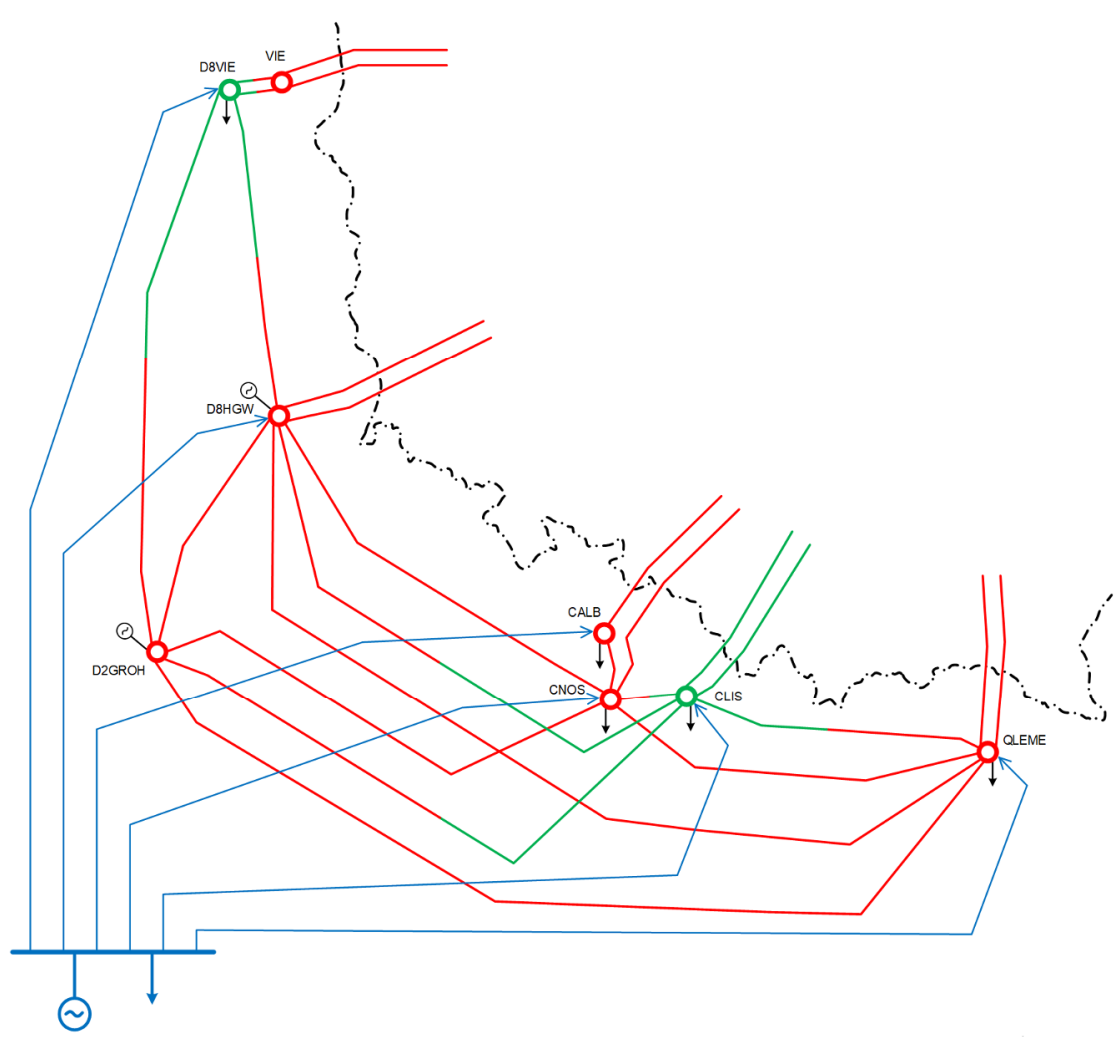

Fig. 3. The equivalent of a foreign network on a synchronous cross-section. 


\section{Use of the trade equivalent}

Below selected cases explaining the operation of the developed model are presented. In these cases. the distribution factors determined above and exemplary values of loop flow (500 MW) and imports (1000 MW) or exports (1000 MW) were adopted.

\subsection{Power exchange on a synchronous cross-section with loop flow}

The first analysed case presents the exchange on a synchronous cross-section at the time of the lack of energy import and export to the NPS. In this situation there is only loop flow in the form of a loop import occurring on the western border and a loop export in the south of the country (Figure 4).

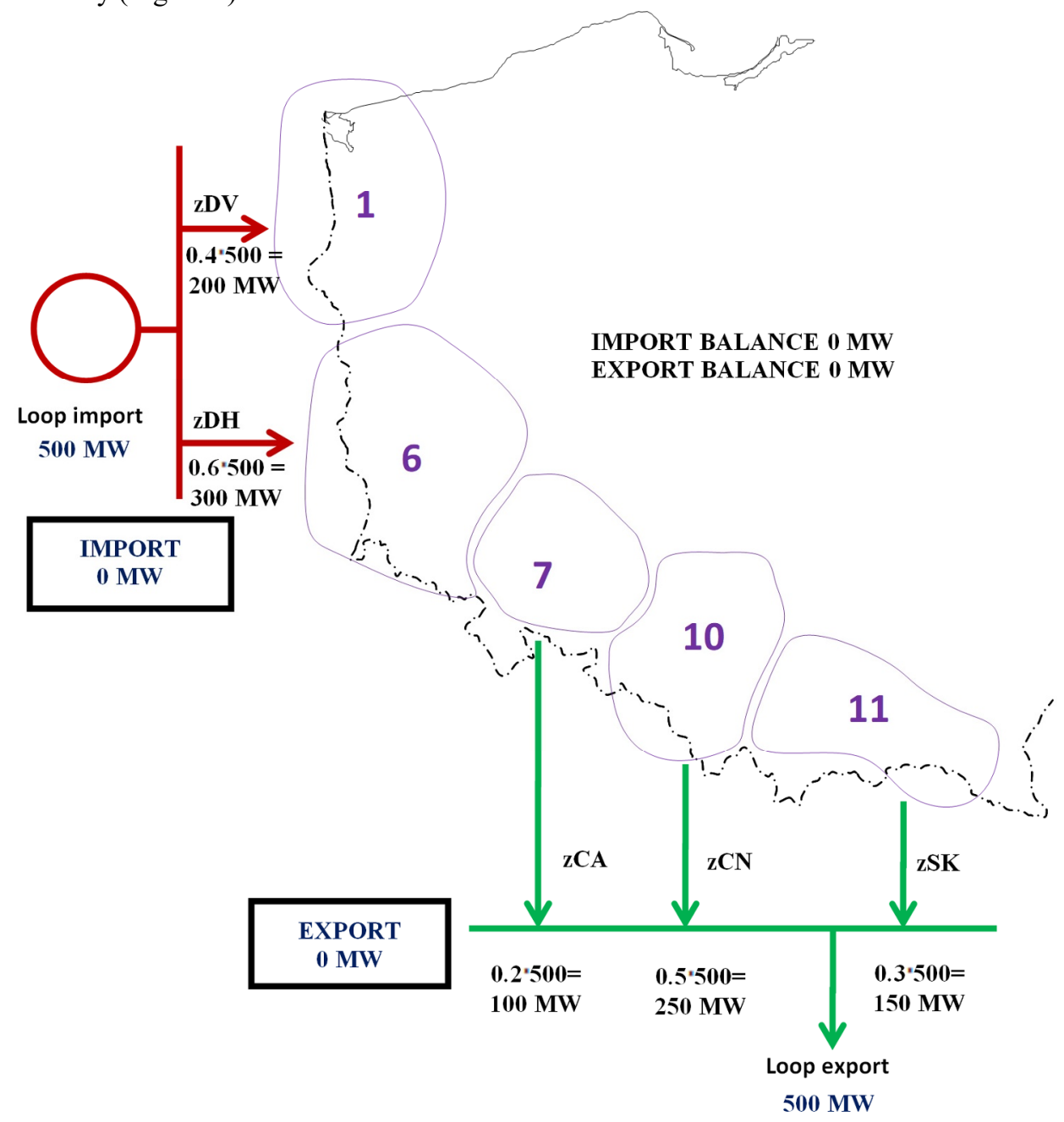

Fig. 4. Power exchange on a synchronous cross-section with loop flow.

In Figure 4500 MW flows from the Germany side into the NPS. which is split between the branches $\mathrm{zDV}$ and $\mathrm{zDH}$ according to the values of the determined coefficients for the $2016 / 17$ winter peak of 0.4 and 0.6 respectively (Table 2 ).

The loop flow balance is determined by the relationship (2) in which 


$$
\text { Loop import }+ \text { Loop export }=0
$$

Accordingly. the total power exchange on the NPS synchronous cross-section with loop flow is 0 :

$$
200 \mathrm{MW}+300 \mathrm{MW}-100 \mathrm{MW}-250 \mathrm{MW}-150 \mathrm{MW}=0
$$

\subsection{Power exchange on a synchronous section with separated import and loop flow}

The case concerns the power exchange on the NPS synchronous cross-section at the moment of import and loop flow. It covers the situation. where the total loop import is 500 MW and the loop export is $500 \mathrm{MW}$ together with the simulation of import of $1000 \mathrm{MW}$ (Figure 5).

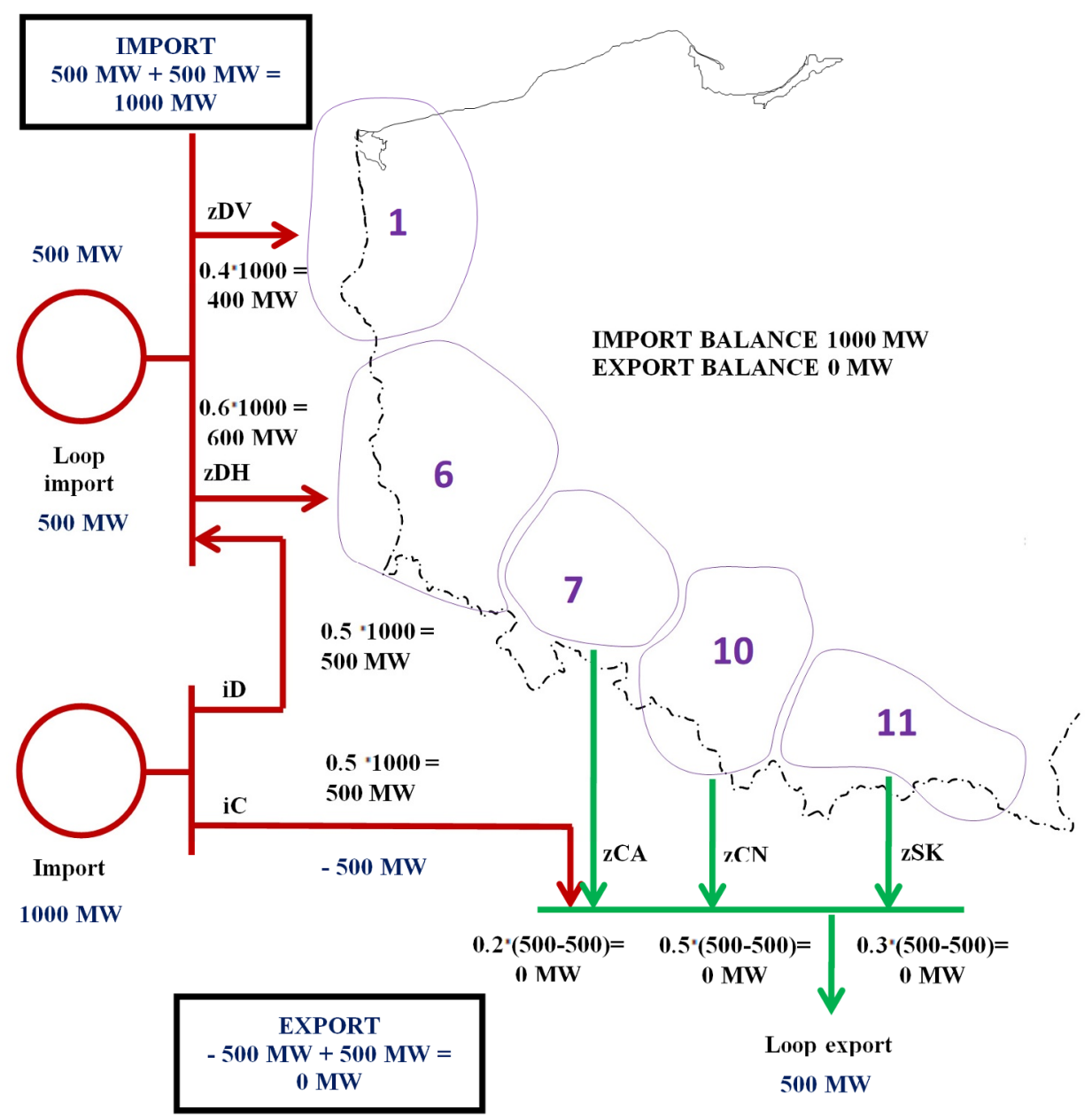

Fig. 5. Power exchange on a synchronous section with separated import and loop flow.

Total power import through western and southern borders equals $1000 \mathrm{MW}$. In accordance with the determined coefficients of the $\mathrm{iD}$ and $\mathrm{iC}$ branches. the power of $500 \mathrm{MW}$ flows to both above mentioned borders. Additionally. taking into consideration 
loop flow. the power flow on branches $\mathrm{zDV}$ and $\mathrm{zDH}$ is $400 \mathrm{MW}$ and $600 \mathrm{MW}$ respectively. According to the formula (2) from the NPS. a total of $500 \mathrm{MW}$ flows out. however. taking into account the power flow of -500 MW resulting from the pre-set 1000 MW import. the value of the resultant export is eliminated (equal to 0). As a result. the total exchange balance in the simulation is $1000 \mathrm{MW}$ reflecting the given level of import. Similar simulations can be performed for exchanges with separated exports and loop flows.

\section{Summary}

The presented example of building a network equivalent was used in the work carried out as part of network development analysis. The realities of research conducted in large cooperating systems require both a large range of analyses and workload. Hence. a reduction in computing cost is required by planners. The presented approach allowed to reduce the model without losing the quality of the obtained results. and hence their usefulness and correctness of inference.

\section{References}

1. H. Oh. A new network reduction methodology for power system planning studies. IEEE Trans. Power Syst. vol. 25. no. 2. pp. 677-684. (2010)

2. H. Oh. Aggregation of Buses for a Network Reduction. IEEE Trans. Power Syst. vol. 27. no. 2. pp. 705-712. (2012)

3. A. Papaemmanouil. G. Andersson. On the reduction of large power system models for power market simulations. 17th Power Systems Computation Conference. Stockholm. Sweden. (2011)

4. O. Małyszko. R. Nowakowski. M. Zeńczak. Estimation of equivalent power system impedance in normal conditions. PAK. vol. 53. no. 6. pp. 11-13. (2007)

5. Di Shi. Power System Network Reduction for Engineering and Economic Analysis. $\mathrm{PhD}$ Dissertation. Arizona State University. (2012)

6. H.K. Singh. S.C. Srivastava. A reduced network representation suitable for fast nodal price calculations in electricity markets. IEEE Power Engineering Society General Meeting. vol. 2. pp. 2070-2077. (2005)

7. H. G. Svendsen. Grid model reduction for large scale renewable energy integration analyses. 12th EERA Deepwind R\&D Conference. Trondheim. Norway. (2015)

8. X. Cheng. T. J. Overbye. PTDF-based power system equivalents. IEEE Trans. Power Syst. vol. 20. no. 4. pp. 1868-1876. (2005) 